\title{
Acute generalized exanthematous pustulosis simulating toxic epidermal necrolysis: case presentation and literature review
}

\author{
Ana-Maria Copaescu ${ }^{1 *}$, Danielle Bouffard ${ }^{2}$ and Marie-Soleil Masse
}

\begin{abstract}
Background: Acute generalized exanthematous pustulosis (AGEP) and toxic epidermal necrolysis (TEN) are severe cutaneous adverse reactions. These rare conditions differ in clinical presentation, pathological features, treatment and prognosis, but overlap has been described implying a challenging clinical management.

Case presentation: We describe a case of overlap between TEN and AGEP probably secondary to beta-lactams in a 77-year-old patient treated for a complicated cholangitis. We review the diagnosis and the management of these two conditions. The diagnosis of TEN was suggested by the initial clinical presentation with severe hemodynamic instability, skin detachment, positive Nikolsky sign and mucosal involvement. However, the skin biopsy as well as the rapid improvement of the skin lesions were discriminative for AGEP. This indicated an overlap presentation. Unfortunately, the patient refused allergy investigations in order to find the culprit drug. Medical photographs, proper physical examination and histopathological results are integrated.

Conclusion: Despite clinical features indicating a diagnosis of TEN, histopathology was conclusive for AGEP thus indicating a possible clinical-pathological overlap between the two conditions, a scarcely described situation in the medical literature. To our knowledge, this is one of the few cases that portrays a TEN-AGEP overlap probably secondary to Piperacillin Tazobactam. Understanding the immunological implications of these conditions can help us better distinguish and manage these severe reactions.
\end{abstract}

Keywords: Acute generalized exanthematous pustulosis, Toxic epidermal necrolysis, Severe cutaneous adverse drug reaction, Disease overlap, Beta-lactam antibiotics

\section{Background}

A severe adverse drug reaction is defined as a life threatening response that requires inpatient care or results in persistent disability or incapacity [1]. Toxic epidermal necrolysis (TEN) and acute generalized exanthematous pustulosis (AGEP) are examples of severe adverse cutaneous drug reactions. These conditions differ in clinical manifestations, morphological features,

\footnotetext{
*Correspondence: ana.copaescu@gmail.com

${ }^{1}$ Allergy and Immunology Department, Centre Hospitalier de I'Université de Montréal (CHUM), 264 Boulevard René-Lévesque E, Montréal, QC H2X 1P1, Canada

Full list of author information is available at the end of the article
}

prognosis and treatment, but severe cases of AGEP rarely mimic TEN. The literature describes thirteen cases where the clinical presentation represented a challenge to patient care. The main causal drugs are antibiotics, nonsteroidal anti-inflammatory drugs (NSAIDs), narcotics (morphine), anti-epileptics (carbamazepine), hydroxychloroquine and chemotherapy drugs.

In this report, we describe the case of a 77-yearold female who developed an unusual AGEP-TEN overlap probably secondary to Piperacillin Tazobactam. The patient presented with severe skin detachment, a positive Nikolsky sign, hemodynamic instability and was hospitalized in the burn unit. However, the biopsy was 
consistent with AGEP. We offer a global portrait of this condition including the initial clinical description, the treatment options and the patient's clinical evolution. A discussion and a literature review will follow.

\section{Case presentation}

A 77-year-old female with no known allergies and with a medical history including dyslipidemia, hypertension, hypothyroidism was transferred to our intensive care unit from a community hospital for an important skin eruption. Prior to her transfer, she had been hospitalized for approximately 1 month for cholangitis complicated by sepsis, iatrogenic pancreatitis and portal vein thrombosis. After a chart review, it was noted that she had received Piperacillin Tazobactam for a total of 20 days as well as Ibuprofen as needed. She also had two contrast radiologic exams 5 and 21 days prior to the eruption.

On initial evaluation, the patient was intubated in the context of an important vasoplegic shock with acute kidney failure. She presented with erythema and skin detachment on $80 \%$ and $35 \%$ of her total body surface, respectively, including external genital area but no ophtalmic lesions (Fig. 1). She presented multiple bullae with purulent liquid. With these findings as well as a positive Nikolsky sign on her arms, abdomen and

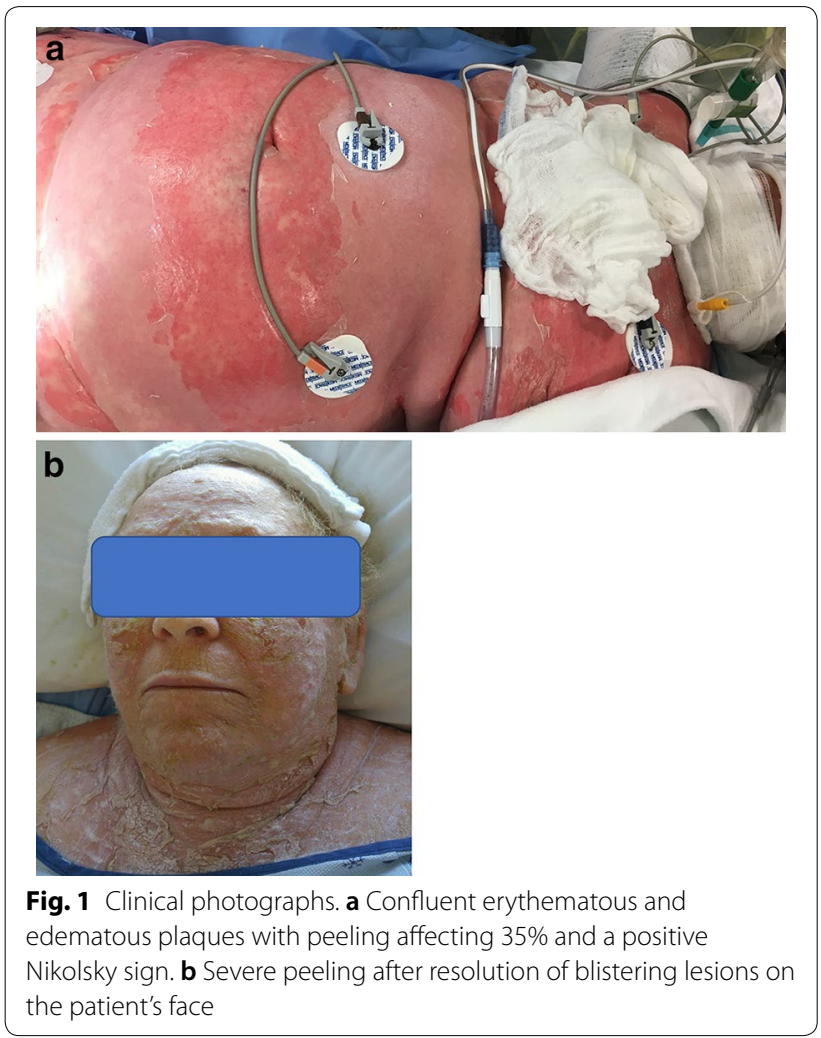

neck region, the most probable diagnosis was toxic epidermal necrolysis (TEN). Her TEN score (Table 1) was calculated at 3 , indicating a very high mortality risk. Twenty-four hours after admission, the patient was sub-febrile (body temperature increased to 38.2 Celsius) and her leukocyte level reached $16.8 \times 10^{9} / \mathrm{L}$.

She was treated with intravenous immunoglobulins $1 \mathrm{~g} / \mathrm{kg} /$ day for a total of 3 days. A skin biopsy from the hand and one from the thigh were obtained upon admission. These biopsies showed sub-corneal pustules with edema of the papillary dermis and an inflammatory infiltrate containing eosinophils. Scattered necrotic keratinocytes were described, but there was no confluent epidermal necrosis. Necrotic keratinocytes were also noted in the intraepidermal portion of a sweat gland (Fig. 2). Direct immunofluorescence was negative. Therefore, the final histopathological diagnosis was compatible with acute generalized exanthematous pustulosis (AGEP).

Several agents were identified as possible culprit: b-lactam antibiotics (Piperacillin Tazobactam), radiologic iodine product and anti-inflammatory drugs (Ibuprofen).

On the 3rd day after admission, she was extubated. After 7 days, no new lesions were noted and the desquamation resumed to less than $5 \%$ of the total body surface. She had an excellent evolution and she was eventually transferred to the referring hospital for rehabilitation.

At 2 months follow-up, the patient was doing well and her skin had healed leaving no scarring. She had the medic-alert bracelet indicating the abovementioned drugs. Unfortunately, the patient refused allergy investigations such as patch testing in order to confirm the culprit drug.

Table 1 SCORTEN scale (reproduced from Bastuji-Garin et al. [28])

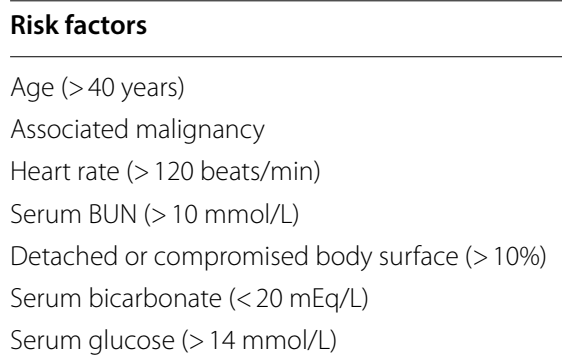

The number of risk factors is correlated with the mortality rate (i.e. for one or less risk factors, the calculated mortality rate is $3.2 \%$, for 2 risk factors, the mortality is $12.1 \%$, for $3,35.3 \%$, for $4,58.3 \%$ and for 5 and more, the mortality rate is more than $90 \%$ 


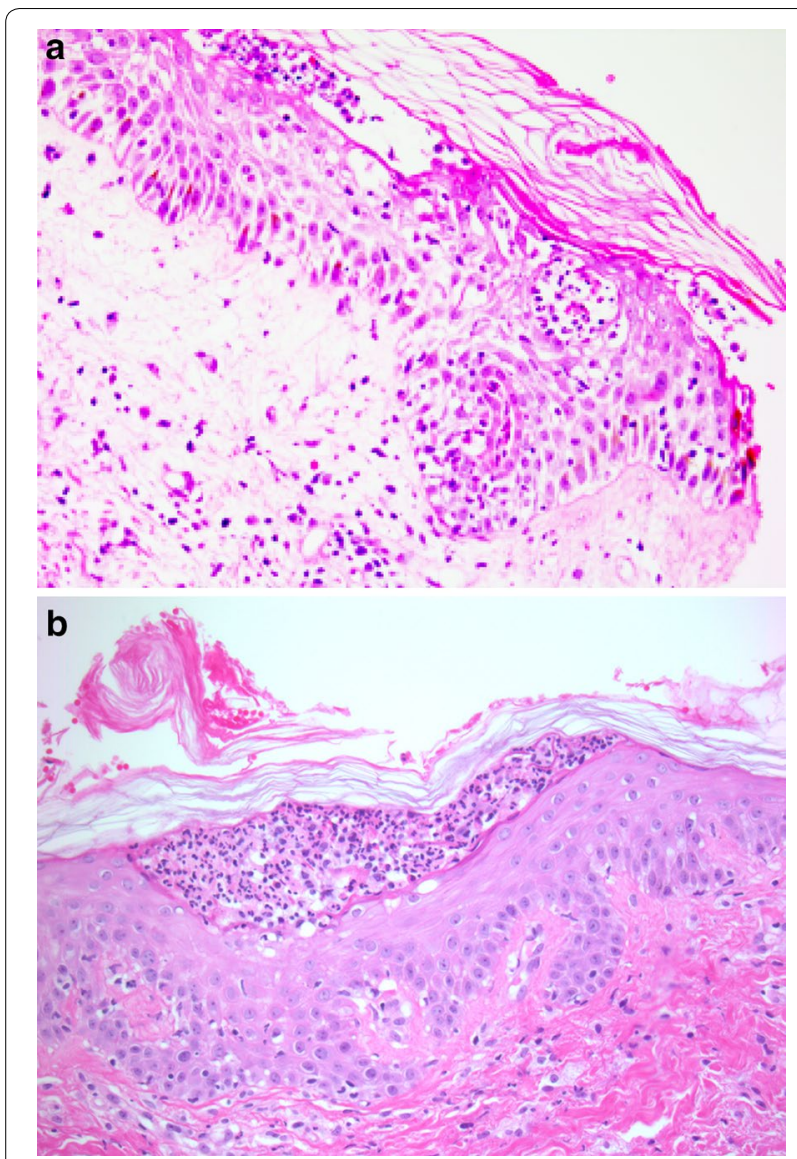

Fig. 2 Histologic picture. a This biospy is from the hand and shows subcorneal pustules with epidermal spongiosis and papillary derma edema. Note the presence of necrosis in the intraepidermal portion of a sweat duct at the edge (hematoxylin and eosin, $\times 20$ ). b This biopsy is from the thigh and shows a subcorneal pustule (hematoxylin and eosin, $\times 20$ )

\section{Discussion and conclusions Better understanding AGEP and TEN}

Both AGEP and TEN are classified as drug reactions. Each clinical entity is the result of an intricate connexion between genetic, immunologic and environmental factors. Their immunological mechanisms are not completely understood but it is well defined that hey both are T-cell-mediated type IV hypersensitivity reactions as portrayed by Gell and Coombs. With increased understanding of the difference between the hypersensitivity reactions included in this group, a subclassification was described according to the pattern of cytokine production by different $\mathrm{T}$ cells and the contribution of certain types of leucocytes. Thus, TEN is a type IVc cytotoxic reaction involving CD8+ cytotoxic killer cells and AGEP is a type IVd reaction that is mainly characterized by the production of neutrophil-attracting chemokine [2]. Research is ongoing to characterize specific cytokines that could play a crucial role in the pathophysiology of AGEP and TEN.

These conditions have very different clinical characteristics. To begin with, the period from starting the culprit drug and the beginning of the skin eruption is about 2 to 3 weeks for TEN, while some AGEP eruptions may begin after a 24-h latency period. The morphological description varies significantly, AGEP being characterised by small pustules on an erythematous background and TEN being recognized by the presence of target lesions, vesicles, bullae, skin detachment with a positive Nikolsky sign and mucosal involvement. In AGEP patients, some authors [3] have described a pseudo positive Nikolsky sign that could represent the coalescence of multiple pustules.

In atypical cases, the biopsy is decisive to formulate the right diagnosis. In our case, the pathologist described subcorneal pustules and edema of the papillary dermis, consistent with AGEP. There have been cases of histological overlap with specific markers of each condition described in the literature [4]. This is not the case of our patient as the pathological features were discriminative for AGEP.

The course of AGEP is usually benign and this condition usually improves after discontinuing the culprit drug. We know that this is not the case for TEN, which is considered life-threatening and can induce multiple long-term complications. These differences and many others are summarized in Table 2.

An interesting observation was formulated by Meiss et al. [5] relating that similar cases of overlap might actually be a two-phase clinical reaction pattern, thus a progression from an AGEP with classic pustules to systemic clinical manifestations characteristic of TEN. Unfortunately, our patient was hospitalized in another center before her hemodynamic instability and thus a complete physical exam before admission is lacking.

A very interesting recent article retrospectively studied Steven-Johnson syndrome/TEN mimickers from four academic hospitals including 208 patients [6]. Out of these patients, $13(6.2 \%)$ had a revised diagnosis of AGEP. The authors concluded that the presence of an atypical target lesion, a positive Nikolsky sign, fever and lymphopenia help predict SIS/TEN.

As described, our patient had a positive Nikolsky sign and was subfebrile. However, no atypical target lesions were described and lymphopenia was absent.

\section{AGEP and TEN overlap-literature review}

As mentioned, both AGEP and TEN are rare skin conditions. Combining both conditions in a patient, either because of the clinical manifestations or the histopathological features is even more rare and we 
Table 2 Description of AGEP and TEN

\begin{tabular}{|c|c|c|}
\hline & AGEP & TEN \\
\hline Incidence & $1-5 /$ million/year & 2-7/million/year \\
\hline Etiology & $\begin{array}{l}\text { Drug }(90 \%) \\
\text { viral, bacterial, or parasitic infections } \\
\text { spider bites }\end{array}$ & $\begin{array}{l}\text { Drug }(60 \%) \\
\text { M. pneumoniae infections } \\
1 / 3 \text { cases no cause }\end{array}$ \\
\hline \multicolumn{3}{|l|}{ Clinical presentation } \\
\hline Distribution pattern & Intertriginous (generalized) & Generalized \\
\hline Mucous Membrane & $20 \%$ (oral) & $100 \%(>30 \%)$ \\
\hline Pustules & Yes & No \\
\hline Target lesions & No & Yes \\
\hline Nikolsky sign & Rare & Yes \\
\hline Fever & Yes & Yes \\
\hline Timing & Hours-days & Days-weeks (<8 wks) \\
\hline Clinical course & Resolution/re-epithelialization 2-4 weeks & \\
\hline Histological features & $\begin{array}{l}\text { Spongiform subcorneal } \\
\text { and/or intraepidermal pustules } \\
\text { edema of the dermis, necrosis of single keratinocytes, and an } \\
\text { inflammatory infiltrate of neutrophils and eosinophils with } \\
\text { perivascular accentuation }\end{array}$ & $\begin{array}{l}\text { Keratinocyte necrosis (partial to full-thickness necrosis of all } \\
\text { epidermis layers) perivascular, discrete lymphohistiocytic, } \\
\text { inflammatory infiltrate (some eosinophils) in the superficial } \\
\text { dermis, } \pm \text { subepidermal bullae }\end{array}$ \\
\hline Prognosis (mortality) & Resolution 2-4 weeks & $\begin{array}{l}\text { Acute phase } 8-12 \text { days } \\
\text { Mortality } 30 \%\end{array}$ \\
\hline Treatment & $\mathrm{d} / \mathrm{c}$ drug & $\begin{array}{l}\mathrm{d} / \mathrm{c} \text { drug } \\
\text { PO or IV corticosteroids, IV immunoglobulin, cyclosporin, } \\
\text { anti-TNF }\end{array}$ \\
\hline
\end{tabular}

found 21 cases described in the literature. In Table 3, we summarize these different cases. It can be noted that there is no tendency towards a specific age group as the patients portrayed are either young adults, middle aged or geriatric patients. There is a slight female predominance in the cases described (14 females and seven males).

As for the culprit drugs questioned, there are several classes of medications but the antibiotics tend to be suspected more frequently with flucloxacillin, a penicillin beta-lactam antibiotic, being on top of the list. In terms of clinical presentation, some cases initially displayed pustules characteristic of AGEP but these skin lesions evolved towards vesicles, bullae and skin detachment with a positive Nikolski sign in a majority of cases. Thus, the patients presented clinical manifestations of TEN but the histopathological examination favored AGEP, with subcorneal spongiform pustules. The clinical evolution and prognosis were more consistent with an AGEP with patients mostly recovering in the first 2 weeks with no residual scarring.

The hemodynamic instability is a feature rarely described in AGEP. Nevertheless, some authors [79] have detailed severe atypical forms of AGEP that presented with systemic inflammatory responses and extensive organ involvement. This form of AGEP might be more frequent in elderly patients with comorbidities.
Although some systemic involvement was described in both AGEP and TEN, the presence of extensive skin detachment requiring intensive care admission and support care is more typical of TEN.

\section{Investigations}

Testing for the causal agent in severe drug reactions remains an area of controversy and the management diverges largely among different regions in the world. Intradermal or patch testing varies in terms of availability, drug concentrations and the use of oral challenges [10]. However, the current literature supports using patch testing in certain specific phenotypes. The method is considered safe with minimal risk of systemic reactions and its sensitivity depends on the culprit drug and the type of non-immediate reaction. Despite the benefits of patch testing in identifying the causal drug, only few articles provide a description of this investigation in cases of overlap [11]. Thus, it can be hypothesised that even though physicians might consider patch testing in classic AGEP patients, the severity of an overlap with clinical features of TEN might discourage the medical team to use this investigational tool. Furthermore, the results from patch testing are intended to help the clinician carry out drug challenges to the negative skin tests results. This is scarcely portrayed. 


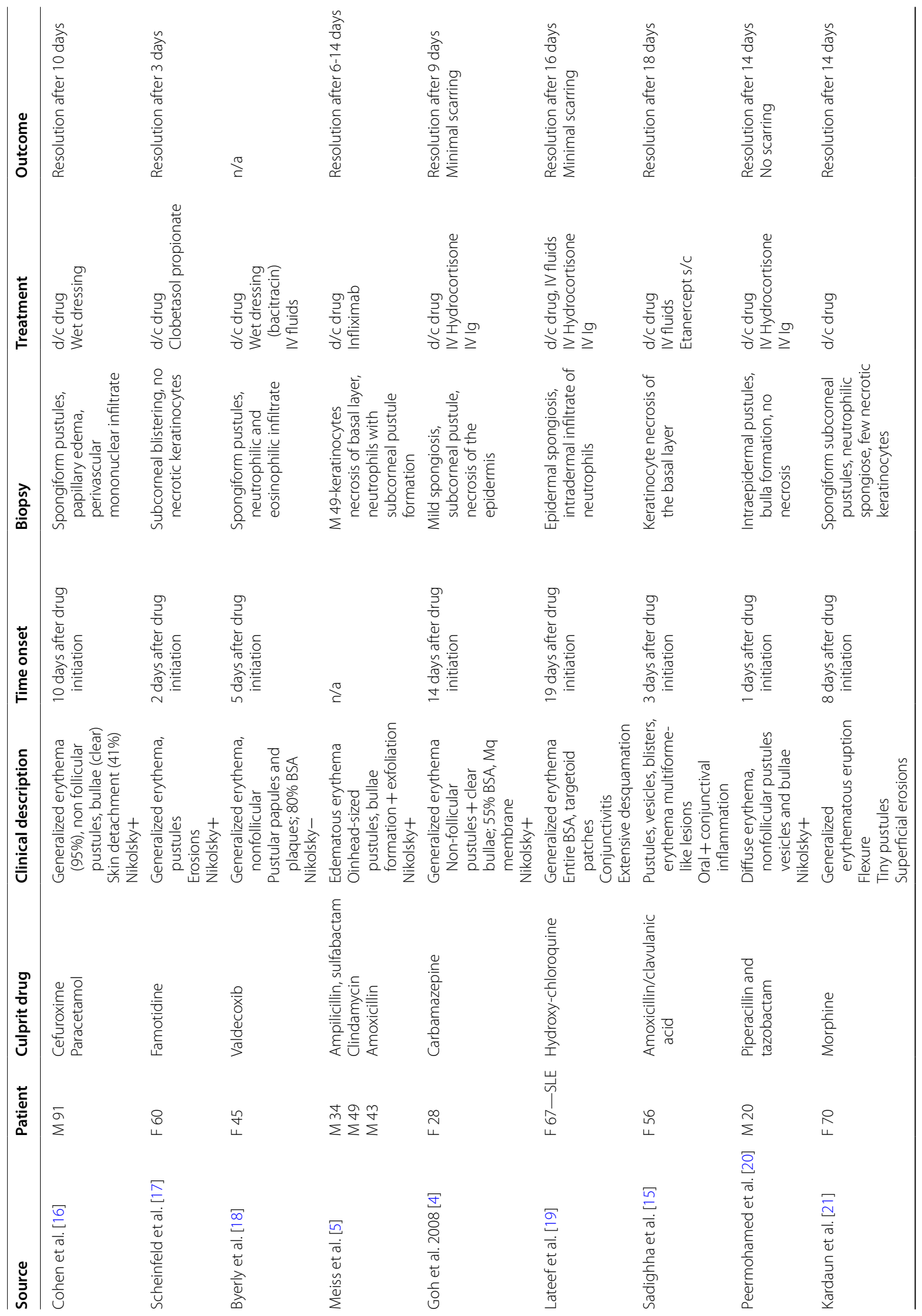




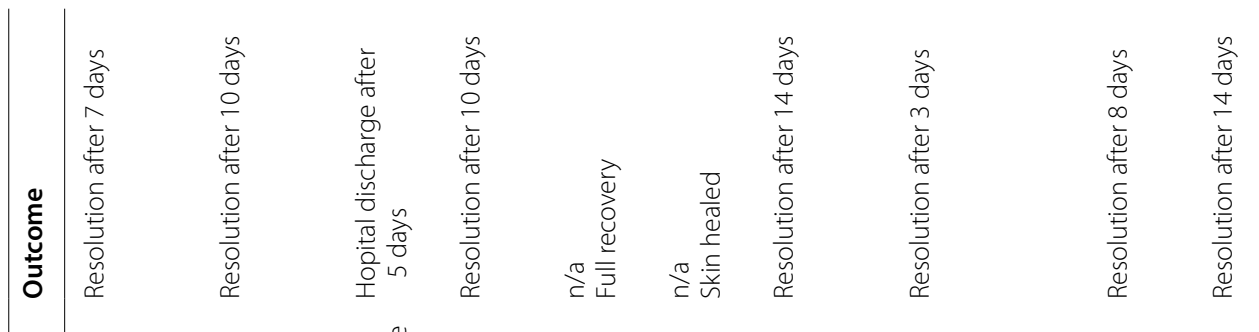

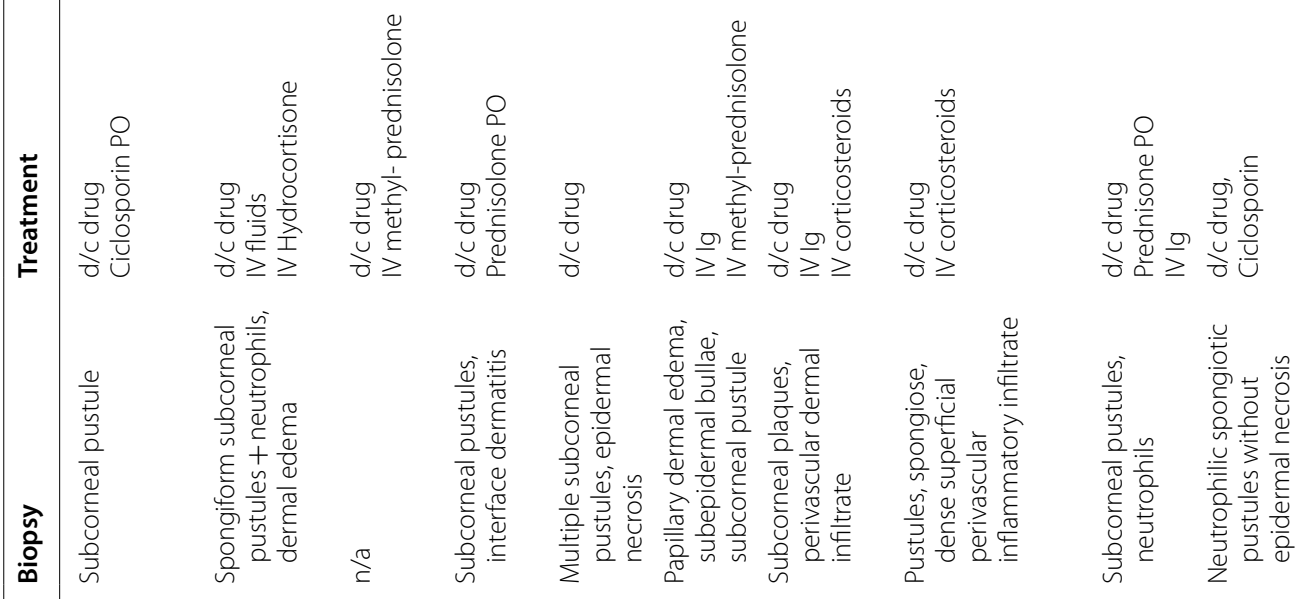

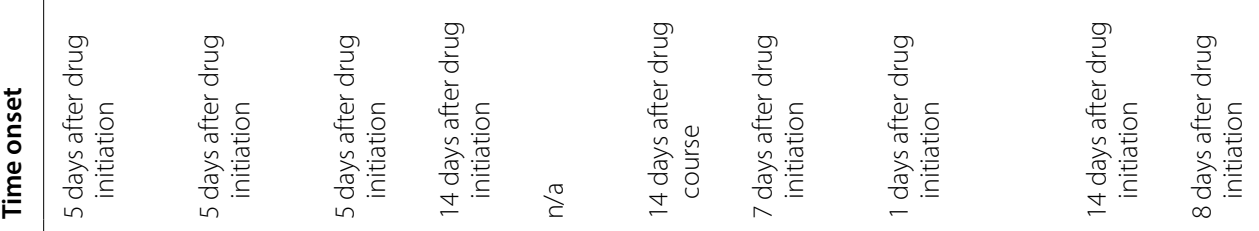
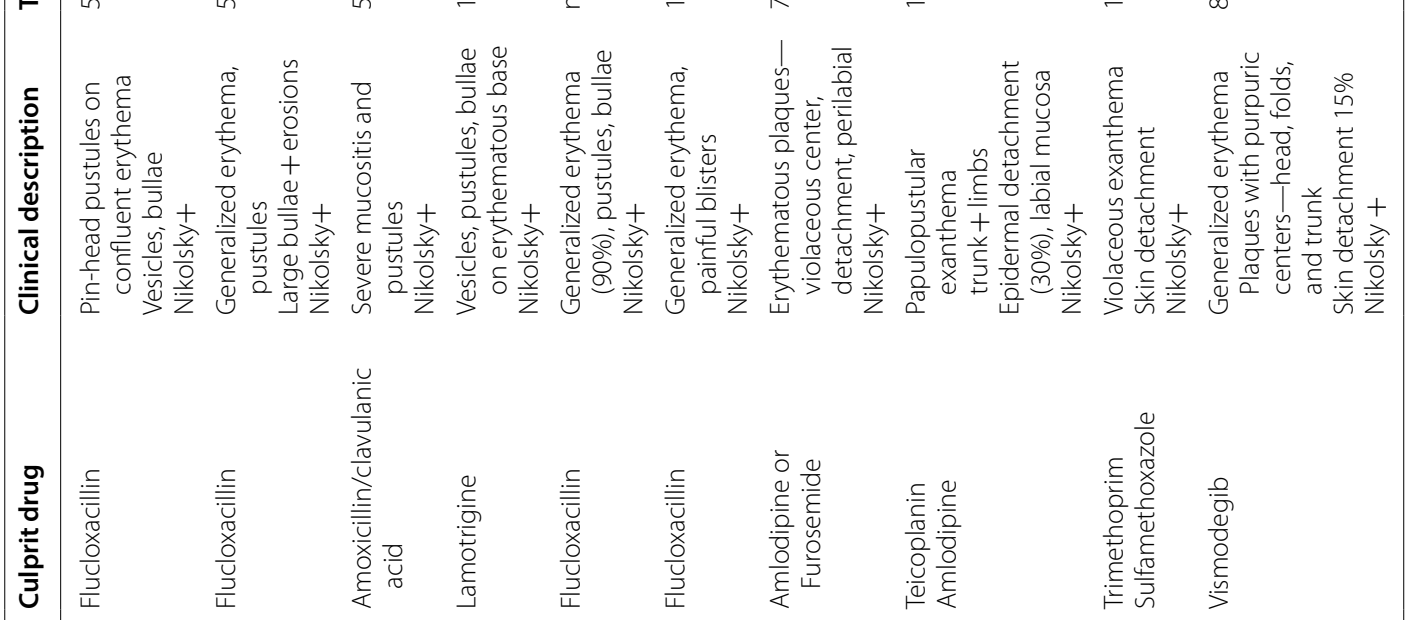

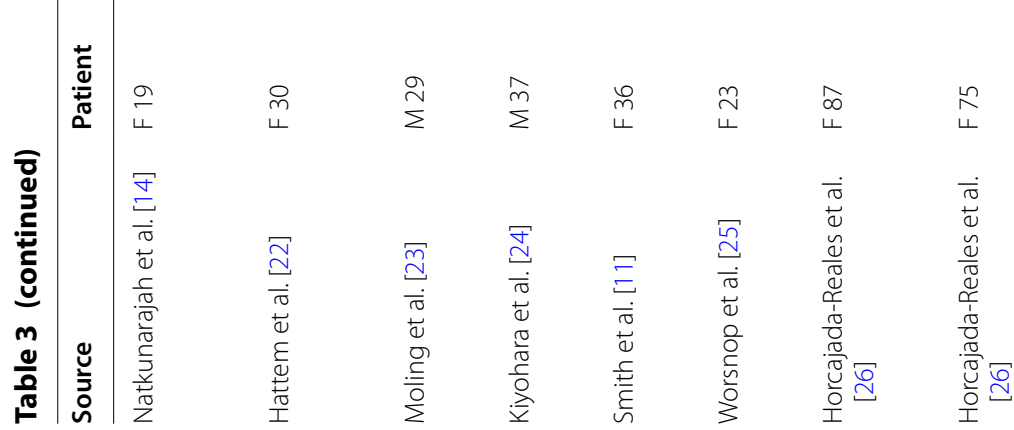

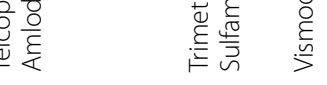


This would have been our approach for this patient if she would have accepted the allergy investigations.

However, novel methods of investigation are on the way. A study from Thailand [12] underlined the importance of detecting drug-specific IFN-y-releasing cells that could help identify up to $46 \%$ of causing agents for SJS/TEN and up to $31.3 \%$ for AGEP.

\section{Management}

Because of the rarity of these severe skin conditions, management is still a source of discussions and research. Authors agree that the first most important step is the withdrawal of the suspected drug and correct identification of the adverse reaction. In patients diagnosed with AGEP, a resolution of the skin eruption is expected within several days after discontinuation of the drug.

In TEN patients, another critical management step is the supportive care fundamental for every burn or intensive care unit patient that presents with severe skin wounds, hemodynamic instability and/or organ failure. Skin care is currently center dependent and there is no evidence to favor the use of debridement, one dressing over another or the correct method to maintain fluid balance [13].

Another favored treatment is the use of corticosteroids as can be observed in the cases of overlap described in the literature. However, their use is still controversial for TEN and data on survival advantage is contradicting. As for AGEP, even if research is further needed, the use of corticosteroids was correlated with a decrease in hospitalization time.

Intravenous immunoglobulins showed no clear benefit in the studies, but no severe side effects either, so clinicians tent to use them quite often for TEN. This is not part of the management for AGEP.

Other case reports $[3,14]$ mention a good clinical response to cyclosporin with rapid resolution of skin eruption.

Meiss [5] has described three cases suggesting an overlap or a two-phase clinical reaction of AGEP and TEN. These patients had high serum tumor necrosis factor (TNF) levels and they responded successfully to a TNF-inhibitor, Infliximab. Further studies are needed to confirm that this success story indicates similar physiopathology in these two conditions.

Another TNF-inhibitor, Etanercept, was used by Sadighha [15] in an overlap case with remarkable results observed only hours after the initial dose as well as rapid cessation of progression of the cutaneous lesions. This author underlines the immunological benefits of using this agent as it decreases a number of cells and cytokines that might play a crucial role in these conditions.

\section{Conclusion}

The initial diagnosis of these skin conditions is based on the clinical presentation. Distinguishing AGEP from TEN allows prompt evaluation and accurate treatment. Our patient presented with severe skin detachment and a positive Nikolsky sign, hallmarks of SJS-TEN. Further pathological investigations and the overall clinical evolution oriented us towards an AGEP diagnosis.

We must ask ourselves if these conditions should actually be described as an overlap presentation or as the manifestation of a severe, aggressive AGEP because of the convincing biopsy?

Further case descriptions and systematic research are need to help us elucidate if these atypical cases are an overlap of the two conditions, a two-phase clinical disease entity of a manifestation of severe AGEP mimicking TEN. We thus encourage clinicians to describe these cases and we believe that an international register of these conditions as well as developing new investigational tools such as blister fluid analysis can help us evaluate this co-presentation of two different immunological processes.

\section{Abbreviations}

AGEP: Acute generalized exanthematous pustulosis; TEN: Toxic epidermal necrolysis; TNF: Tumor necrosis factor; NSAIDs: Nonsteroidal anti-inflammatory drugs.

\section{Acknowledgements}

Not applicable.

\section{Authors' contributions}

MM is the patient's physician and DB is the pathologist consulted to analyze the skin biopsy. AC did the chart and literature review and wrote the manuscript draft. DB and MM contributed to the revision of the manuscript for important scientific content. All authors read and approved the final manuscript.

\section{Funding}

Not applicable.

Availability of data and materials Not applicable.

\section{Ethics approval and consent to participate}

Informed consent for publication was obtained from the patient.

\section{Consent for publication}

The patient was informed that de-identified data would be used in the scientific research and publications. After a detailed discussion and answering all the patient's questions, informed consent for publication was obtained from the patient.

Competing interests

The authors declare that they have no competing interests. 


\author{
Author details \\ ${ }^{1}$ Allergy and Immunology Department, Centre Hospitalier de I'Université \\ de Montréal (CHUM), 264 Boulevard René-Lévesque E, Montréal, QC H2X \\ 1P1, Canada. ${ }^{2}$ Pathology Department, Centre Hospitalier de I'Université de \\ Montréal (CHUM), Montréal, Canada.
}

Received: 25 July 2019 Accepted: 22 January 2020

Published online: 04 February 2020

\section{References}

1. Nebeker JR, Barach P, Samore MH. Clarifying adverse drug events: a clinician's guide to terminology, documentation, and reporting. Ann Intern Med. 2004;140(10):795-801.

2. Bellon T. Mechanisms of severe cutaneous adverse reactions: recent advances. Drug Saf. 2019;42:973-92. https://doi.org/10.1007/s40264-01900825-2.

3. Moreno-Arrones $\mathrm{OM}$, et al. Acute generalized exanthematous pustulosis simulating Stevens-Johnson syndrome/toxic epidermal necrolysis associated with the use of vismodegib. JAAD Case Rep. 2018;4(2):123-5.

4. Goh TK, et al. Acute generalised exanthematous pustulosis and toxic epidermal necrolysis induced by carbamazepine. Singap Med J. 2008:49(6):507-10.

5. Meiss F, et al. Overlap of acute generalized exanthematous pustulosis and toxic epidermal necrolysis: response to antitumour necrosis factor-alpha antibody infliximab: report of three cases. J Eur Acad Dermatol Venereol. 2007:21(5):717-9.

6. Weinkle A, Pettit C, Jani A, Keller J, Lu Y, Malachowski S, et al. Distinguishing Stevens-Johnson syndrome/toxic epidermal necrolysis from clinical mimickers during inpatient dermatologic consultation-a retrospective chart review. J Am Acad Dermatol. 2019;81(3):749-57. https ://doi.org/10.1016/j.jaad.2019.05.061

7. Tajmir-Riahi A, et al. Life-threatening atypical case of acute generalized exanthematous pustulosis. Int Arch Allergy Immunol. 2017:174(2):108-11.

8. De Coninck AL, et al. Acute generalized exanthematous pustulosis induced by paracetamol. A case with severe hemodynamic disturbances. Dermatology. 1996;193(4):338-41.

9. McDonald KA, Pierscianowski TA. A case of amoxicillin-induced acute generalized exanthematous pustulosis presenting as septic shock. J Cutan Med Surg. 2017;21(4):351-5.

10. Phillips EJ, et al. Controversies in drug allergy: testing for delayed reactions. J Allergy Clin Immunol. 2019;143(1):66-73.

11. Smith VM, et al. Acute generalized exanthematous pustulosismimicking toxic epidermal necrolysis in patients with psoriasis. Clin Exp Dermatol. 2016:41:557-67.

12. Klaewsongkram J, et al. Analysis of HLA-B allelic variation and IFN-gamma ELISpot responses in patients with severe cutaneous adverse reactions associated with drugs. J Allergy Clin Immunol Pract. 2019;7(1):219-227.e4.

13. Cho YT, Chu CY. Treatments for severe cutaneous adverse reactions. J Immunol Res. 2017;2017:1503709.

14. Natkunarajah J, Ostlere L. Severe acute generalized exanthematous pustulosis with blistering, mimicking toxic epidermal necrolysis. Clin Exp Dermatol. 2012;37(2):188-9.
15. Sadighha A. Etanercept in the treatment of a patient with acute generalized exanthematous pustulosis/toxic epidermal necrolysis: definition of a new model based on translational research. Int J Dermatol. 2009;48(8):913-4.

16. Cohen AD, Cagnano E, Halevy S. Acute generalized exanthematous pustulosis mimicking toxic epidermal necrolysis. Int J Dermatol. 2001:40(7):458-61.

17. Scheinfeld N, et al. Acute generalized exanthematous pustulosis resembling toxic epidermal necrolysis caused by famotidine. Acta Dermato-Venereologica. 2002;83:76-7.

18. Byerly FL, et al. Valdecoxib-associated acute generalized exanthematous pustulosis. Burns. 2005;31(3):383-7.

19. Lateef A, Tan KB, Lau TC. Acute generalized exanthematous pustulosis and toxic epidermal necrolysis induced by hydroxychloroquine. Clin Rheumatol. 2009;28(12):1449-52.

20. Peermohamed S, Haber RM. Acute generalized exanthematous pustulosis simulating toxic epidermal necrolysis: a case report and review of the literature. Arch Dermatol. 2011;147(6):697-701.

21. Kardaun $\mathrm{SH}$. Acute generalized exanthematous pustulosis (AGEP), presenting with toxic epidermal necrolysis-like features, due to morphine: a neglected culprit? Eur J Dermatol. 2011;21(3):427-8.

22. van Hattem S, Beerthuizen GI, Kardaun SH. Severe flucloxacillin-induced acute generalized exanthematous pustulosis (AGEP), with toxic epidermal necrolysis (TEN)-like features: does overlap between AGEP and TEN exist? Clinical report and review of the literature. Br J Dermatol. 2014;171(6):1539-45

23. Moling O, Perino F, Piccin A. Acute generalized exanthematous pustulosis with overlap features of toxic epidermal necrolysis/Stevens-Johnson syndrome. Int J Dermatol. 2014;53(1):e27-8.

24. Kiyohara T, et al. Toxic epidermal necrolysis with some features of acute generalized exanthematous pustulosis. Acta Dermato-Venereologica. 2013;93:212-3.

25. Worsnop F, et al. Acute generalized exanthematous pustulosis mimicking toxic epidermal necrolysis in patients with psoriasis: a coincidence? Clin Exp Dermatol. 2015;40(6):688-9.

26. Horcajada-Reales C, Pulido-Perez A, Suarez-Fernandez R. Severe cutaneous drug reactions: do overlapping forms exist? Actas Dermosifiliogr. 2016;107(1):23-33.

27. García Abellán J, et al. Acute generalized exanthematous pustulosis simulating toxic epidermal necrolysis: overlapping processes. Actas Dermosifiliogr. 2018;109:460-2.

28. Bastuji-Garin S, Fouchard N, Bertocchi M, Roujeau JC, Revuz J, Wolkenstein P. SCORTEN: a severity-of-illness score for toxic epiderma necrolysis. J Invest Dermatol. 2000;115(2):149-53. https://doi.org/10.104 6/j.1523-1747.2000.00061.x

\section{Publisher's Note}

Springer Nature remains neutral with regard to jurisdictional claims in published maps and institutional affiliations.

Ready to submit your research? Choose BMC and benefit from

- fast, convenient online submission

- thorough peer review by experienced researchers in your field

- rapid publication on acceptance

- support for research data, including large and complex data types

- gold Open Access which fosters wider collaboration and increased citations

- maximum visibility for your research: over 100M website views per year

At BMC, research is always in progress.

Learn more biomedcentral.com/submissions 